\title{
VARIATION DES CARACTÉRISTIQUES DES FIBRES DE LAINE, SELON LES REGIONS DU CORPS CHEZ LE MOUTON
}

PAR

\author{
P. CHARLET, A. M. LEROY et P. CATTIN-VIDAL
}

Laboratoire de Recherches Zootechniques de l'Institut National Agronomique ${ }^{(1)}$

\section{PLAN DU MEMOIRE}

\section{I. - Introduction. \\ II. - Conditions de cette étude. \\ III. - Interprétation des résultats.}

\section{INTRODUCTION}

Les toisons étant constituées par un nombre considérable de fibres dont les caractéristiques sont fort différentes, il ne peut être question, pour étudier une toison, que de procéder à l'étude d'échantillons judicieusement choisis.

La détermination des points où 1'on doit prélever les échantillons qui permettront d'estimer au mieux la valeur des caractéristiques de la toison dans son ensemble nécessite auparavant une connaissance précise de la variation de ces caractéristiques selon la région d'implantation.

L'appréciation rapide des qualités d'une toison, par un ou plusieurs prélèvements, est intéressante à plusieurs titres.

I Elle permet seule de caractériser une toison en vue d'études de sélection ou d'hérédité.

$2^{\circ}$ Elle permet de suivre l'influence du milieu (alimentation, etc...) sur ces caractéristiques.

\section{CONDITIONS DE CETTE ÉTUDE :}

Cette étude de la topographie des caractéristiques des fibres sur une même toison, a été effectuée sur 7 brebis Ile-de-France, non en lactation, et maintenues en parfait état d'entretien.

(1) Avec la collaboration technique des Etablissements de la Chambre de commerce de Roubaix (laboratoire d'analyses et de recherches industrielles) et la participation financière du Fonds national du Progrès agricole (crédits en provenance du Fonds d'encouragement à l'industrie textile, mis à notre disposition sur l'initiative de la Fédération nationale ovine). 


\section{Détermination des points de prélèvement}

Sur chacune de ces brebis, les points de prélèvement ont été déterminés par l'intersection de lignes, tracées préalablement sur les animaux et partant de points de repère osseux, faciles à déterminer et fixes dans leur position. Au total, 38 échantillons par animal.

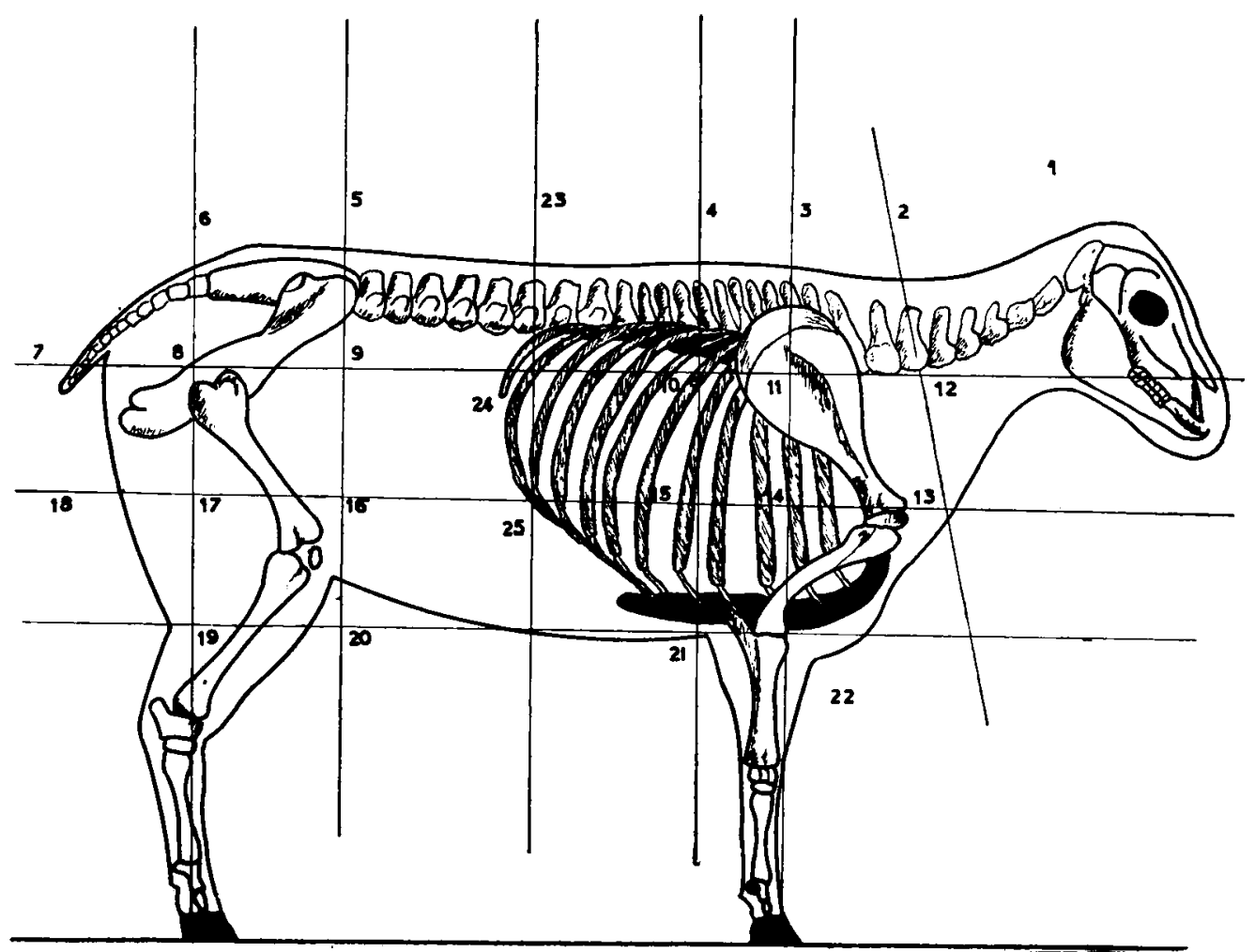

FIG. I.

Ces points de repère fixes nous ont permis d'établir des "cartes" précises à l'échelle de la toison (voir fig. I et 2).

\section{Mesures effectuées}

Sur chacun des échantillons nous avons effectué les mesures suivantes :

Io Détermination de la proportion de fibres textiles lavées à fond, à partir de l'échantillon brut.

$2^{\circ}$ Mesure de la longueur moyenne des fibres.

$3^{\circ}$ Mesure de leur finesse moyenne et indice d'homogénéité de cette finesse. 


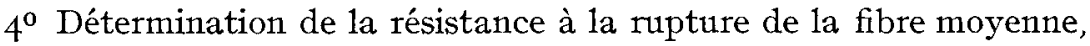
de sa résistance à l'unité de section, et de son élasticité.

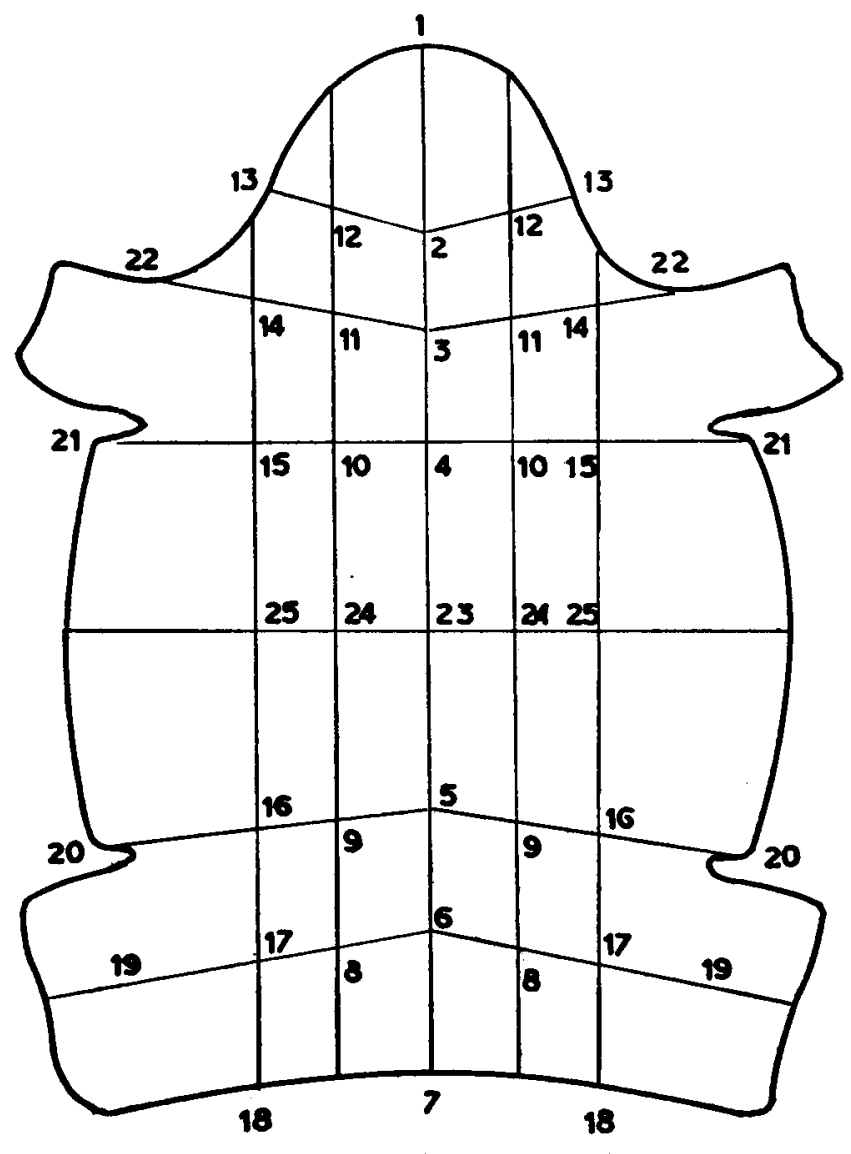

FIG. 2.

Ligne $1,2,3,4,23,5,6,7:$ Ligne du dessus.

Ligne $7,8,9,24$, I0, I I, I 2 : Ligne horizontale au niveau de la pointe du trochanter.

Ligne I3, I4, 15, 25, I6, 17, I8: Ligne horizontale au niveau de l'articulation scapulo-humérale.

Ligne I9, 20: Horizontale au niveau du dessous du ventre.

Ligne 2, I2, I3: Périmètre de la base du cou.

Ligne 3, I I, I4 : Verticale du garrot.

Ligne 4, Io, I5, 2 I : Périmètre thoracique au passage des sangles.

Ligne $23,24,25$ : Verticale issue de l'insertion de la dernière côte.

Ligne $5,9, \pm 6,20$ : Verticale de l'ilium au grasset.

Ligne 6, 8, I7, I9: Verticale du trochanter.

$5^{\circ}$ Poids de laine et nombre de brins à l'unité de surface par une méthode directe mise au point au laboratoire (Annales de l'I.N.R.A., $\mathrm{n}^{0} \mathrm{I}$ de I952, p. I47 à I56). 


\section{MÉTHODES EMPLOYÉES}

ro Proportions de fibres textiles lavées à fond à partir de l'échantillon brut.

Nous pesons exactement chaque échantillon brut après conditionnement à $65^{\circ}$ d'hygrométrie et I $8^{\circ}$ de température. Ensuite, les échantillons sont successivement lavés à 1'eau distillée tiède $\left(25^{\circ}\right)$, lavés avec une solution contenant $80 \mathrm{~g}$ de $\mathrm{CO}_{3} \mathrm{Na}_{2}$ par litre d'eau distillée, préalablement chauffée à $40-45^{\circ}$, rincés à l'eau chaude $\left(45^{\circ}\right)$, passés sous un jet d'eau froide pour enlever l'excès de savon ; finalement nous plongeons chacun des échantillons dans 3 bains successifs de trichloréthylène technique, en déplaçant les coupelles et en renouvelant le dernier bain à chaque échantillon.

Ensuite, séchage pendant I 2 heures dans une étuve à $45^{\circ}$, puis conditionnement $(65 \%$ et $18 \%)$.

Une deuxième pesée nous permet de déterminer la proportion de laine propre, par rapport à l'échantillon brut.

\section{$2^{\circ}$ Mesures de la longueur des brins}

La longueur moyenne de chaque échantillon a été déterminée par les méthodes suivantes :

a) Pour 3 des 7 brebis :

Mesure de la longueur de roo brins tirés un à un et au hasard à la base de chaque échantillon.

b) Pour les 7 brebis :

Le poids d'une mèche, divisé par le poids d'une portion de longueur connue de cette dernière, prise en son milieu, donne la longueur moyenne de la mèche.

On utilise la cisaille Stutz, qui tranche une portion de $2 \mathrm{~cm}$ dans la mèche, d'où

$$
\begin{array}{ll}
\mathrm{L}=2 \frac{\mathrm{P}}{\mathrm{p}} & \mathrm{L}=\text { longueur moyenne de la mêche } \\
\mathrm{P} & =\text { poids de la mêche } \\
p & =\text { poids de la portion de la mêche de } 2 \mathrm{~cm} .
\end{array}
$$

Cette méthode est plus rapide, mais ne permet pas de mesurer 1'homogénéité des longueurs dans la mèche (mèches plus ou moins pointues). La parfaite concordance des 2 séries de résultats de 3 brebis nous a permis d'adopter la seconde méthode pour mesurer les longueurs des autres brebis, ce qui a permis un gain de temps appréciable.

\section{$3^{\circ}$ Mesure de la finesse des fibres}

Mesure directe du diamètre des brins vus au microscope à projection, après avoir peigné et écarté la mèche prise dans des pinces larges (genre pince à dessin) pour isoler les brins (50 lectures). 
Un autre laboratoire a effectué les mesures de la finesse des 3 pre. mières brebis en opérant de la même façon, mais en faisant too lectures-

Les deux séries de résultats concordent dans l'ensemble. Quelques valeurs différentes nous ont amenés à refaire une troisième mesure en utilisant, cette fois, la méthode gravimétrique, où l'on calcule le diamètre moyen des fibres, à partir de leur poids, leur longueur et leur densité, supposée égale à $\mathrm{I}, 3 \mathrm{I}$ *.

\section{3 bis Indice d'homogénéité des finesses}

Seules, les mesures par lecture directe des diamètres permettent de calculer cet indice.

Il s'obtient en appliquant la formule

$$
\sigma=\sqrt{\frac{e^{2} f}{n-1}} \quad \begin{aligned}
& e=\text { écarts à la moyenne } \\
& t=\text { fréquences } \\
& n=\text { nombre de lectures. }
\end{aligned}
$$

$4^{0}$ Résistances et élasticités

L'élasticité et la résistance à la rupture ont été mesurées simultanément à l'aide du dynamomètre Richard, qui enregistre graphiquement l'allongement du brin de laine au moment de la rupture en même temps que la charge de rupture exprimée en grammes. Un jeu de formules appropriées permet de calculer la résistance à la rupture par unité de surface de section du brin. Pour obtenir une bonne mesure, il convient de répéter ces opérations sur Io brins, en choisissant ceux-ci de manière que leur diamètre moyen soit aussi voisin que possible de celui de l'échantillon à analyser.

$$
5^{\circ} \text { Tassé }
$$

Des prélèvements ont été faits sur les 4 derniers animaux à l'aide de l'appareil utilisé par l'un de nous et décrit dans l'étude " Méthode d'appréciation directe du tassé des toisons".

Dix-sept échantillons par animal, chacun représentant $\mathrm{I} / 4{\mathrm{de} \mathrm{cm}^{2}}^{2} \mathrm{de}$ surface de peau, ont été pesés et comptés.

\section{INTERPRÉTATION DES RÉSULTATS}

Io Pour chaque série de résultats (finesse, longueur, etc...) nous avons reporté les chiffres bruts sur les "cartes " aux points de prélèvements correspondants.

$2^{0}$ Pour délimiter sur les cartes, les diverses zones, nous avons déter-

$\left(^{*}\right)$ Le Mouton, par André M. LERoY, 19ł8. Librairie IIachette. 
miné graphiquement les points où les caractéristiques ont une valeur entière sans décimales. La méthode utilisée était celle des valeurs proportionnelles :

Soit à déterminer sur le graphique le point oì les filtres doivent mesurer 27 microns.

On choisit les points voisins où les finesses des filtres ont des valeurs décimales de part et d'autre de la valeur entière considérée.

Par exemple: point $n^{\circ}$ Io, $26,2 \mu$;

$$
\text { point } n^{\circ} 15,27,6 \mu \text {. }
$$

L'écart entre ces valeurs est de 1,4 !..

L'écart entre la finesse du point $n^{0}$ Io et la finesse de valeur entière est 0,8 .

Le point où la finesse est de valeur entière $27 \mu$ sera situé à partir du point $n^{0}$ Io sur la droite reliant le point $n^{0}$ Io au point $n^{0}$ I5 à une distance égale à $\frac{0,8}{\mathrm{I}, 4}$ de la distance totale IO.I5.

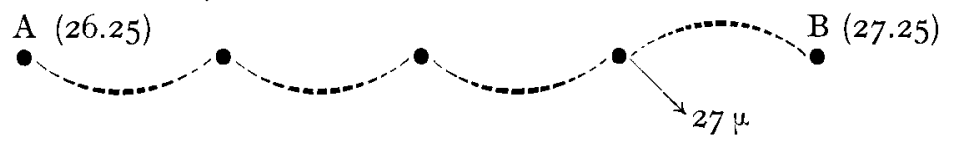

$3^{0}$ Aux mêmes points de valeur entière les caractéristiques ont été exprimées en pour cent de la valeur moyenne de la toison.

$4^{\circ}$ Les moyennes des résultats exprimés en pour cent pour un même point de prélèvement, nous ont permis d'établir des cartes représentant la variation des caractéristiques pour un animal moyen en délimitant des zones précises.

\section{I $^{\circ}$ Proportion de fibres textiles lavées à fond à partir de l'échantillon brut}

Ces proportions varient avec les points de prélèvement, mais auss avec les sujets. D'une façon générale, cette proportion diminue aux endroits souillés (arrière-train, ventre, côté sur lequel l'animal se couche).

Le milieu du dos retient souvent aussi des impuretés.

Aucune variation systématique ne semble établie.

\section{$2^{\circ}$ Longueur des fibres}

Les variations sont plus régulières d'un sujet à l'autre; la toison moyenne nous montre que :

- Les mèches les plus longues se trouvent à la cuisse ainsi que sur la pointe du sternum de l'animal.

- Les mèches les plus courtes sont sur le cou et sur le ventre; pour ce demier point, on peut penser que, lorsque l'animal se couche, il y a usure des fibres; cependant, les cuisses de l'animal, également soumises à cette usure, présentent les fibres les plus longues. 


\section{Répartition des Longueurs}

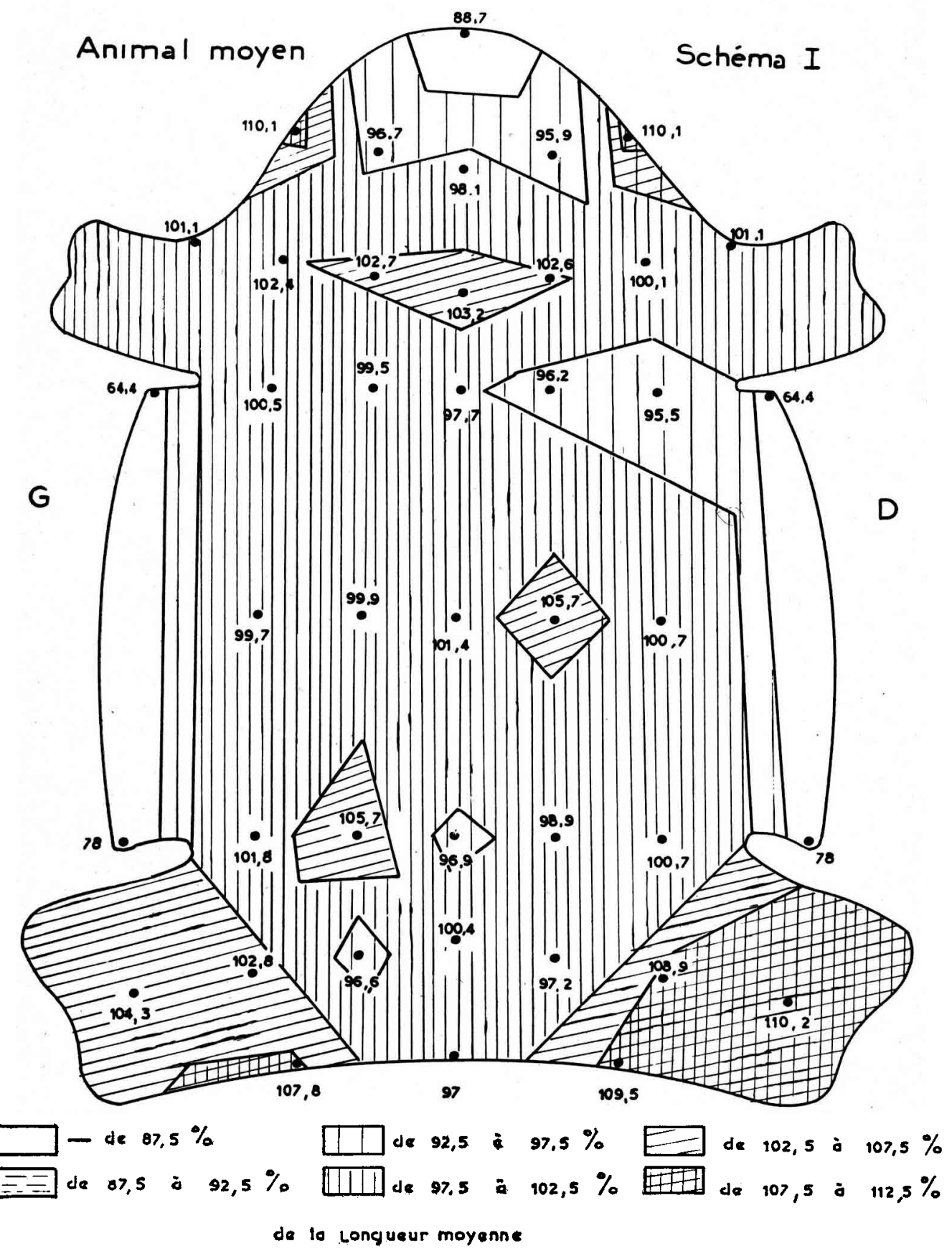

Les indications de ce tableau et des tableaux suivants se rapportent aux quotients des valeurs mesurées en chaqus point par la valeur moyenne de l'ensemble de la toison, multipliée par 100. 
- L'épaule et le dos des animaux ont une longueur toujours très proche de la moyenne. Cependant, les fibres du dessus de la croupe tendent à être légèrement plus courtes (Schéma I).

\section{$3^{\circ}$ Finesse des brins}

Ici, les variations restent leș mêmes chez les 7 animaux. Seule l'étendue des régions à fibres plus fines ou plus grossières varie avec les sujets, suivant l'homogénéité de leur finesse dans la toison.

- Les mèches les plus fines se trouvent au niveau du cou et sur la partie haute de l'épaule, de chaque côté du garrot.

- Les mèches les plus grossières sont surtout sur 1'arrière-train, avec un maximum à l'arrière de la cuisse.

- Les finesses moyennes se rencontrent sur les flancs et sur le milieu du dos, en une zone très étendue constituant ainsi un point de prélèvement intéressant (Schéma II).

La répartition des différentes finesses, présente une analogie avec celle des longueurs ainsi que l'indique la corrélation définie plus loin.

\section{$4^{\circ}$ Résistance}

La résistance ne semble pas suivre de variations constantes avec la région de la toison. Toutefois, les flancs semblent souvent se rapprocher de la moyenne (Schéma III).

$$
5^{\circ} \text { Tassé }
$$

La densité d'implantation varie assez régulièrement d'un animal à l'autre.

Les épaules, et surtout le dos, sont les régions du corps les plus tassées.

Le cou et les flancs se situent dans la moyenne alors que les cuisses ont un tassé très inférieur à la moyenne (Schéma IV).

\section{Relations entre les différentes caractéristiques des fibres de la toison}

Io Pour chaque animal, il existe une relation très étroite entre la finesse et l'indice d'homogénéité de cette finesse. Dans la toison, les mèches les plus fines sont aussi les plus homogènes. La corrélation est de :

$$
\begin{array}{ccc}
\text { Brebis } & n^{\circ} \mathrm{I}+0,75 \\
" & n^{\circ}+0,67 \\
" & n^{\circ} 3+0,78 \\
" & n^{\circ} 4+0,65 \\
" & n^{0} 5+0,52 \\
" & n^{0} 6+0,42 \\
" & n^{0} 7+0,56
\end{array}
$$




\section{Répartition des Finesses}

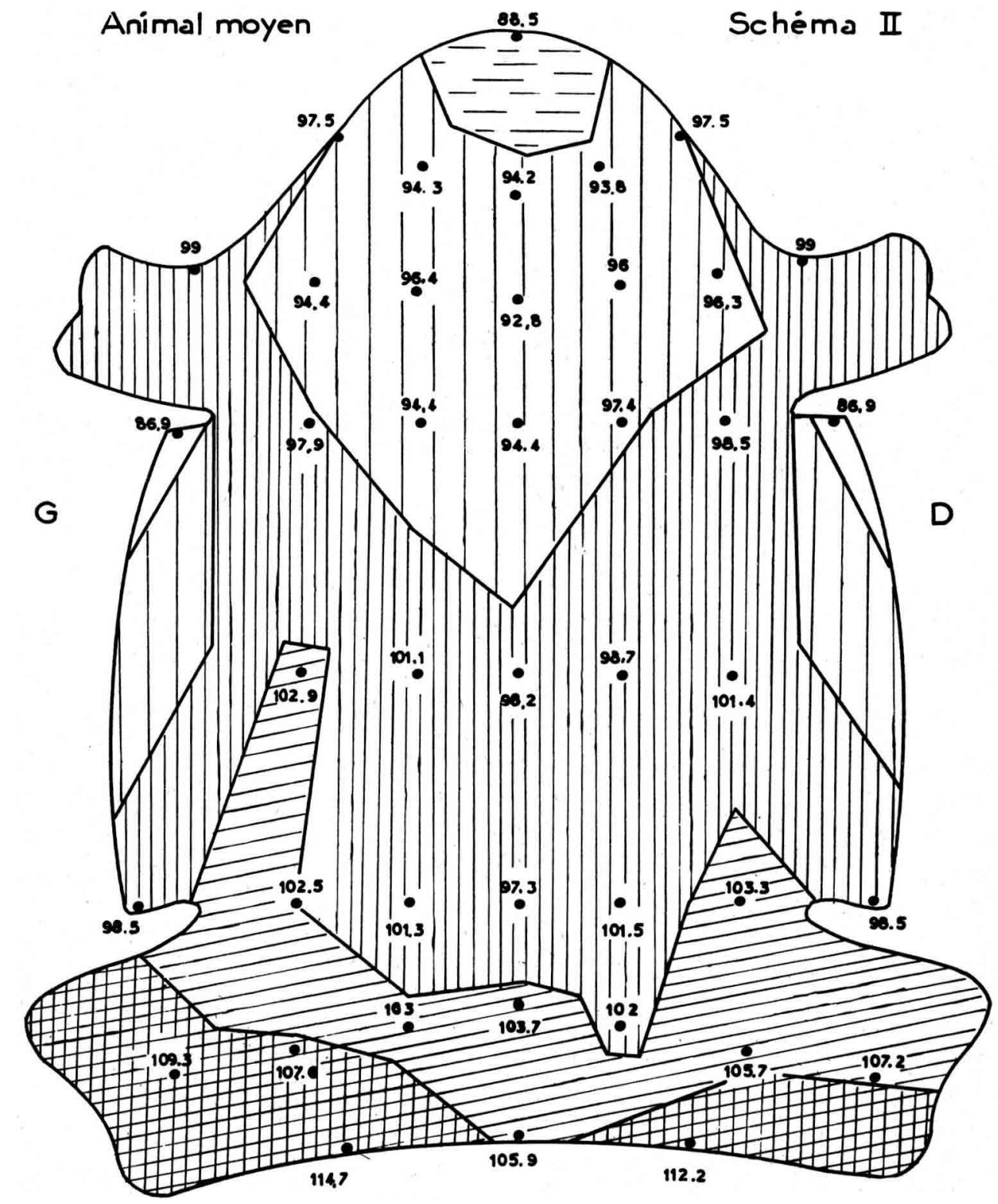

$\square$ - de $07,5 \%$

$\square$ de 92,5 ò $97,5 \%$ de 102,5 ì $107,5 \%$ 三 de 07,5 ò $22,5 \%$ पill de 97,5 a $102,5 \%$ de $107,5 \%$ du diamétre moyen 
I 86

P. CHARLET, A.-M. LEROY ET P. CATTIN-VIDAL (II, I953)

Répartition de la résistance

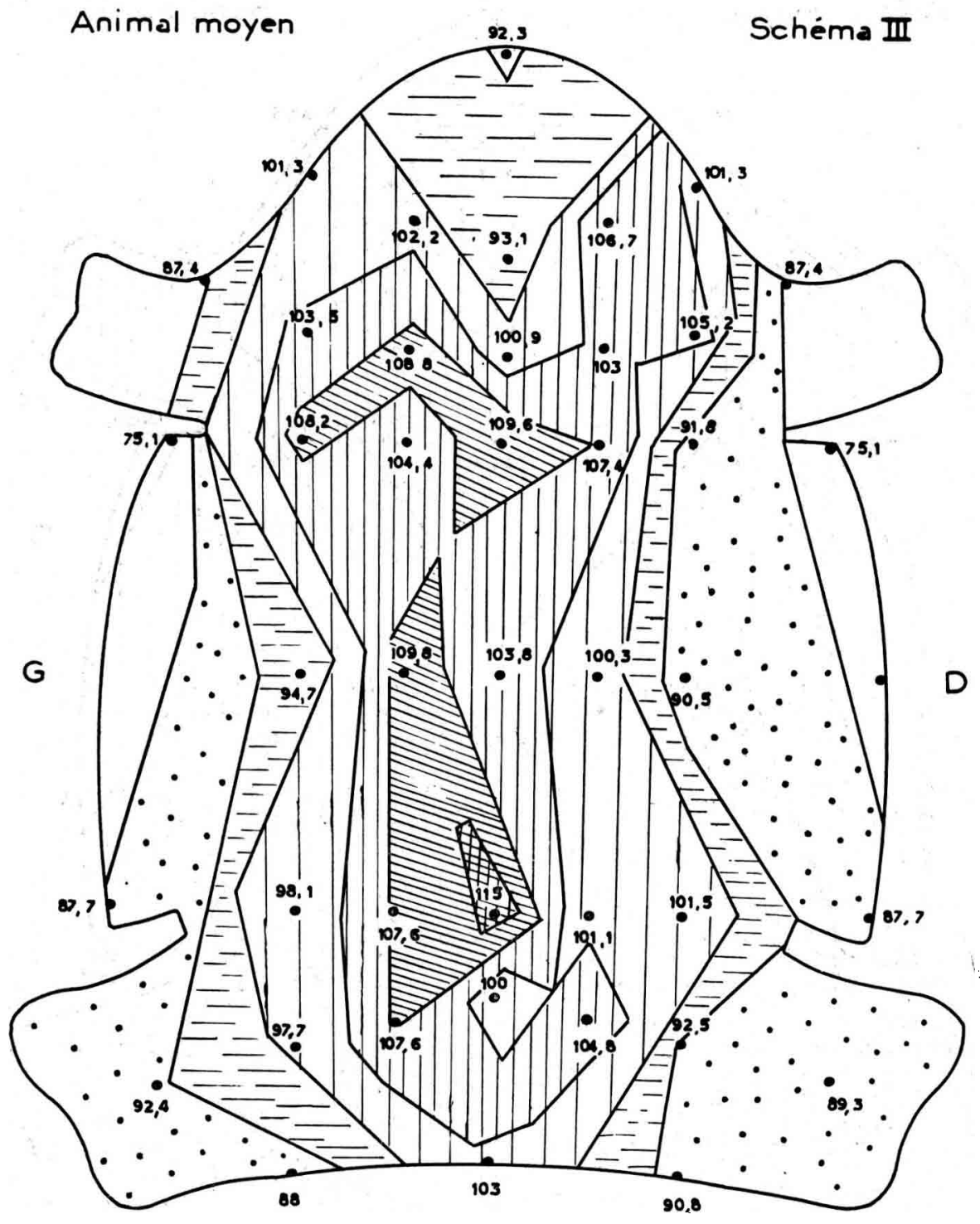

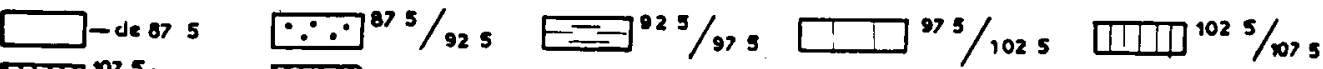
I1075 1125 


\section{Répartition du Tassé}

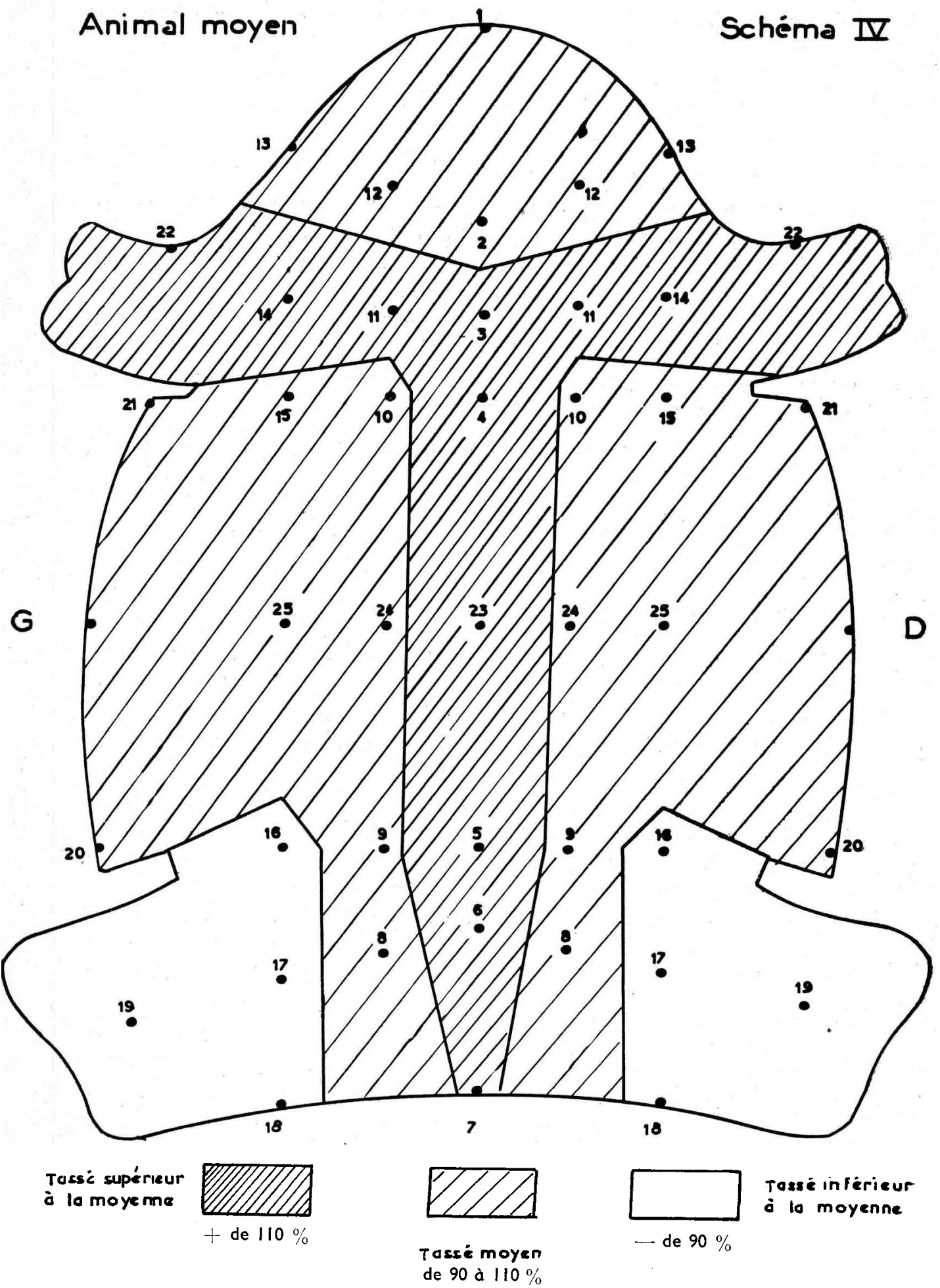


$2^{\circ}$ La relation entre le diamètre des fibres et leur résistance à l'unité de section, est de :

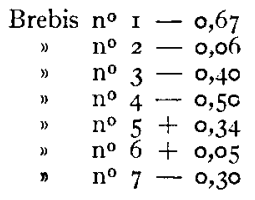

Ainsi, nous constatons, sur 4 brehis $(\mathrm{I}-3-4-7)$ que plus les diamètres sont élevés plus la résistance à l'unité de surface est faible.

Deux brebis ne présentent pas de corrélation (2-6). Cette corrélation pour la brebis 5 , est positive. La corrélation diamètre/résistance au micron carré de la brebis moyenne est de - o,I5, ce qui montre tout de même une tendance à l'abaissement de la résistance à l'unité de section quand les diamètres augmentent. Cette tendance est en accord avec ce que l'on sait de la structure du brin de laine.

$3^{\circ}$ La relation entre le diamètre des fibres et leur longueur est de :

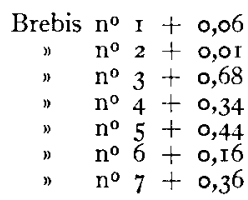

Les brebis $3-4-5-6-7$, dont la corrélation est nettement positive, présentent des brins de laine qui sont d'autant plus longs que le diamètre est plus élevé.

Les brebis I et 2 présentent une relation pratiquement nulle.

Pour 1'animal moyen, la corrélation diamètre/longueur est de $+0,57$, ce qui est significatif.

Il ressort de cette étude que l'appréciation d'une toison pourrait être faite dans la race Ile-de-France :

I $^{\circ}$ A l'aide d'un prélèvement au niveau du flanc (point no 25 sur le schéma) où toutes les caractéristiques y sont moyennes, à l'exception toutefois de la répartition des résistances qui semble varier d'un animal à l'autre d'une manière, pour le moment, impossible à expliquer.

$2^{\mathrm{o}}$ A l'aide de 2 prélèvements, dont la moyenne donne la valeur moyenne de la toison:

a) A l'épaule (en arrière de l'articulation scapulo-humérale, point $n^{\circ}$ I4).

b) A la cuisse (à mi-distance entre le trochanter et 1'articulation fémoro-rotulienne) (point intermédiaire entre les $\mathrm{n}^{0}$ I6 et I7).

Cette deuxième méthode possède l'avantage de donner une idée de 1'homogénéité de la toison, pour les différentes caractéristiques : finesse, longueur, résistance et tassé, ce qui est d'une très grande importance pour la valeur de la production lainière de l'avenir. 